\title{
Design of Hybrid Technique based on RGB Contents and Entropy of the Image and its Comparative Analysis with Existing Technique
}

\author{
Rajeev Sardana \\ Head, Comp. Engg. Dept. \\ Govt. Polytechnic, Hisar
}

\author{
Maitreyee Dutta, Ph.D \\ Professor \& Head, CSE Dept. \\ NITTTR, Chandigarh.
}

\author{
Surender Singh Saini \\ Sr. Scientist, Optical Devices \& \\ Systems \\ CSIR-CSIO, Chandigarh.
}

\begin{abstract}
Image fusion is finding its application in all spheres of life like medical, military, geological etc. The aim of Image fusion is to combine the information of same scene images taken from different sensors or the images with focus on different objects. Fused image is more informative and better in quality. In this paper, a hybrid technique is developed and carried out a detailed comparative analysis with the existing techniques based on RGB contents, Entropy of the image. This paper is based on considering the remote sensing application
\end{abstract}

\section{General Terms}

Image fusion, PCA, Wavelet Transforms, Hybrid Technique.

\section{INTRODUCTION}

The goal of Image fusion is to combine the information of same scene images taken from different sensors or the images with focus on different objects. Fused image is more informative and better in quality. Image fusion techniques are normally used to enhance the applications of the source images, improve reliability and capability of the images. The fused image has better information than source image which is useful for human or machine applications. The fused image with rich information not only improve the performance of image analysis algorithms but also its applications. A feature closely related to image quality is the focus. Sharp images provide better information than blurry images. However, in some situations it is not possible to obtain totally focused images in just one single camera shot, since some regions appear to be blurred due to variations in the depth of the scene and because of the camera lenses focus. This means that if the camera is focused at one specific object, another region of the scene can be out of focus. An interesting solution is to take more pictures of the desired landscape in the same position, but with focus centered in different elements of the scenery. Then, using the image fusion concept, all source images are combined, creating a single image that contains all the best focused regions. Usually, data in image and video processing problems are described in the spatial or time domain, but other domains (e.g. frequency domain) can turn their representation and/or analysis more convenient. Fused Image can be extracted using several methods broadly classified in spatial domain and transform domain (Transform the image to its frequency representation).

Principal Component Analysis (PCA), Intensity Hue Saturation (IHS) and High Pass filtering methods fall in spatial domain fusion techniques. Spatial image fusion work by combining the pixel values of the two or more images. On the other hand, Wavelet Transform and Laplacian Transform methods fall in the transform domain. In transform domain method the multiscale decomposition of the images is done and the composite image is constructed by using the fusion rule. The inverse multiscale transform is applied to achieve the fused image.
The objective of this paper is implementation of fusion techniques (PCA, Wavelet Transform and High Pass filtering approach) and design of a hybrid technique based on these three fusion techniques for better result as paper is based on considering the remote sensing application. The comparison of each technique is carried out with the developed hybrid fusion technique for the different parameters, such as RGB content and Entropy.

\section{IMAGE FUSION}

This section describes the image fusion by Principal Component Analysis (PCA) [7] and Wavelet Transform techniques.

\subsection{Principal Component Analysis (PCA)}

The Principal Component Analysis (PCA) is a mathematical procedure that changes the potentially correlated variables into an uncorrelated variables called principal components. The aim of PCA is to reduce the variables of the dataset with maintaining the original variability in data. Data set is computed in this as a compact and optimal manner. The first principal component presents maximum variance in the data as possible and each succeeding component presents as much of the remaining variance as possible. First principal component is taken to be in the direction with the maximum variance. The second principal component constrained to lie in the subspace perpendicular of the first \& so on. A Principal Component Analysis basis vector depends on the data set.

Algorithm for PCA fusion technique:

Source images (images to be fused) arranged in two-column vectors. For project of this data into a $2-D$ subspaces, following steps are followed:

1. Organise the data into column vectors. The resulting matrix $\mathrm{Z}$ of dimension $2 \mathrm{x} \mathrm{n}$.

2. Empirical Mean along each column is computed. The Empirical Mean vector (ME) of dimension $1 \times 2$.

3. Subtract the empirical mean vector ME from matrix $\mathrm{X}$ is of dimension $2 \times \mathrm{n}$.

4. Covariance matrix $\mathrm{C}$ of $\mathrm{X}$ s calculated i.e. $\mathrm{C}=\mathrm{XX}$ Tean of expectation $=\operatorname{cov}(\mathrm{X})$

5. Now the eigenvectors (V) and eigenvalue (D) of Covariance matrix (C) are computed and sorted by decreasing eigenvalue. Eigenvectors (V) and eigenvalue (D) are of dimension $2 \times 2$.

6. To compute P1 and P2 first column of eigenvectors (V) which corresponds to larger eigenvalue are considered as:

$$
\mathrm{P}_{1}=\frac{\mathrm{V}(1)}{\sum \mathrm{V}} \quad \mathrm{P}_{2}=\frac{\mathrm{V}(2)}{\sum \mathrm{V}}
$$


The fused image will be:

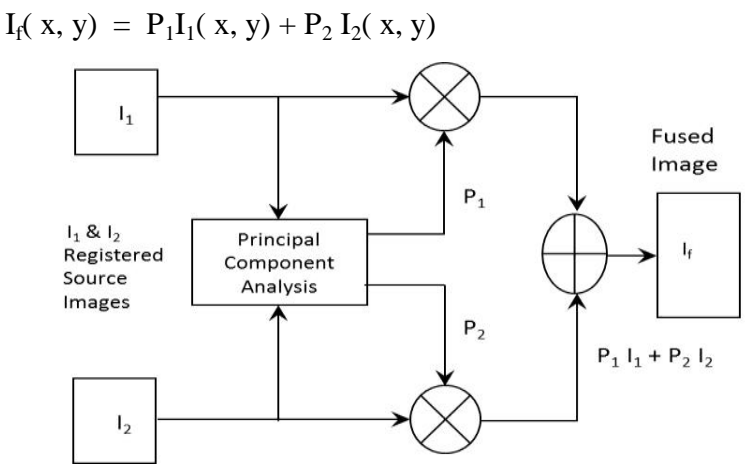

Fig. 1: PCA fusion technique

\subsection{Wavelet Transform}

The wavelet transform is a mathematical tool in which local features of a signal process can be detected. It is used in many applications such as data compression, feature detection, texture analysis and image fusion. A common wavelet transform technique used for fusion is the Discrete Wavelet Transform (DWT). The DWT can be interpreted as signal decomposition in a set of independent, spatially oriented frequency channels. The signal is passed through Low pass \& High pass filters and emerges as two signals, approximation and details. This is called decomposition. Without loss of information these signals can be assembled back into the original signal. This process is known as reconstruction. The mathematical manipulation, which implies analysis and synthesis, is called DWT and inverse DWT. An image can be decomposed into a sequence of different spatial resolution images using DWT. In case of a 2D image, an $\mathrm{N}$ level decomposition can be performed resulting in $3 \mathrm{~N}+1$ different frequency bands namely, LL, LH, HL and $\mathrm{HH}$.

\begin{tabular}{|c|c|c|}
\hline LL2 & HL2 & \multirow{2}{*}{$\begin{array}{c}\text { HL1 } \\
\text { Horizontal }\end{array}$} \\
\cline { 1 - 2 } LH2 & HH2 & $\begin{array}{c}\text { HH1 } \\
\text { Diagonal }\end{array}$ \\
\hline \multicolumn{2}{|c|}{$\begin{array}{c}\text { LH1 } \\
\text { Vertical }\end{array}$} & \\
\hline
\end{tabular}

Fig. 2: Level 2 Wavelet Decomposition

Algorithm for wavelet fusion technique

1. Input two multi-focus images. Here in our proposed algorithm we have considered two images which are of same size (registered images).

2. Apply Discrete Wavelet Transform on both the images.

3. Extracts from the discrete wavelet decomposition structure, the horizontal, vertical, diagonal \& Approximation detail.

4. Perform max. of approximation \& mean of horizontal, vertical and diagonal coefficients of both decomposed images. Perform this for all the pixel values of image i.e. $\mathrm{m}$ $\mathrm{x}$ n. Thus we will get $\mathrm{HH}_{\mathrm{n}}, \mathrm{HL}_{\mathrm{n}}, \mathrm{LH}_{\mathrm{n}}$ and $\mathrm{LL}_{\mathrm{n}}$ as new coefficients.

5. Take Inverse Discrete Wavelet Transform.

6. Obtain the fused Image and Display.

The fused image $\mathrm{I}_{\mathrm{f}}(\mathrm{x}, \mathrm{y})$ is obtained by taking the inverse of the discrete wavelet transform as:
$\left.\mathrm{I}_{\mathrm{f}}(\mathrm{x}, \mathrm{y})=\operatorname{IDWT}_{\mathrm{DW}} \boldsymbol{\boldsymbol { \Phi }}\left\{\mathrm{DWT}\left(\mathrm{I}_{1}(\mathrm{x}, \mathrm{y})\right), \operatorname{DWT}\left(\mathrm{I}_{2}(\mathrm{x}, \mathrm{y})\right)\right\}\right]$

The fusion rule used in this paper is simply averages the approximation coefficients.

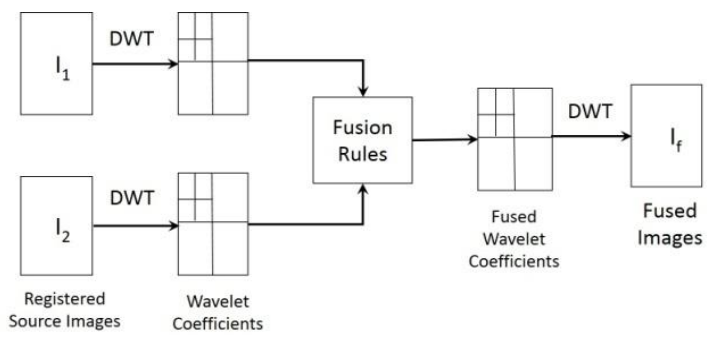

Fig. 3: Wavelet fusion technique

\section{PROPOSED HYBRID TECHNIQUE}

The image fusion is very useful technique for merging information of multiple sensors. Different algorithms have been proposed which produced good results but also yields some problems, such as Brovey fused image is very dark \& hard to identify the ground features [5]. In PCA technique, there is spectral distortion in fused image because it does not extract a component representing spatial detail from the image. IHS loses certain spectral information [1]. HPF image is very fuzzy and has very bad visual effect. Also, the edges are emphasized too much \& produce spectral distortion [2]. Wavelet Transform fused images produce good spectral preservation but poor spatial improvement [4]. Hence good fusion techniques is required for better result. The final fused image has a good spatial resolution. To overcome these limitations we propose a new technique combining PCA fusion technique, Wavelet fusion technique \& high pass filter approach known as Hybrid Technique.

Algorithm for Hybrid Fusion Technique

1. Input two multi-focus images

2. Rescale the images as per requirement.

3. Apply Discrete Wavelet fusion technique on images.

4. Apply High Pass filter to images.

5. High-spatial contents extracted from the images using highpass filter are concatenated.

6. Fused image obtained after applying wavelet technique \& High spatial contents of the images after concatenation are fused together using PCA technique.

7. Finally the fused image is obtained

Calculate the desired parameters of the fused image.

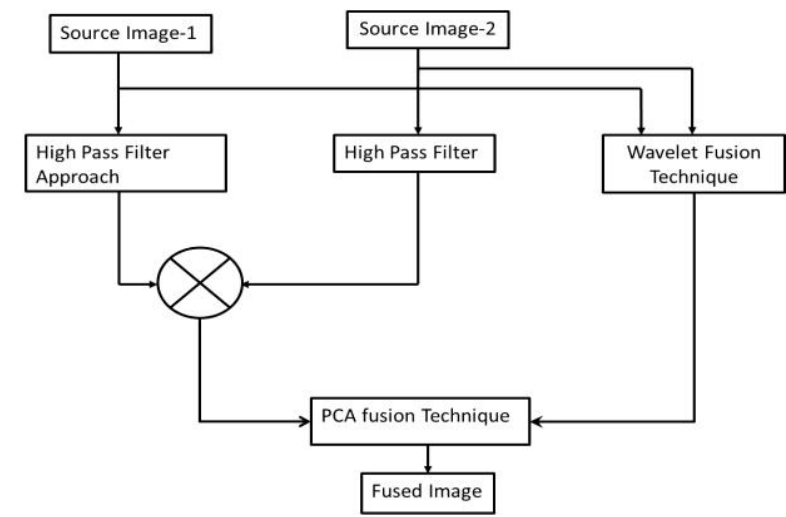

Fig. 4: Proposed Hybrid Fusion Technique 


\section{EXPERIMENTAL RESULTS, VISUAL OBSERVATION AND COMPARATIVE ANALYSIS}

In this section we present our experiments on two sets of original \& blurred images to evaluate the image fusion algorithms. The image set contains one original image \& two it's blurred. In first, images are blurred on bottom \& upper portion. In second, Images are blurred on left bottom corner \& right upper corner.

On these images PCA, DWT, Proposed Hybrid fusion algorithms are applied \& fused image along with required parameters (RGB contents, Entropy) are obtained. Results are compared based on these parameters.

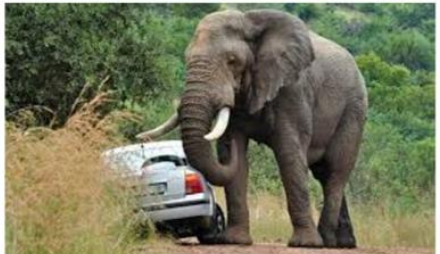

(a)

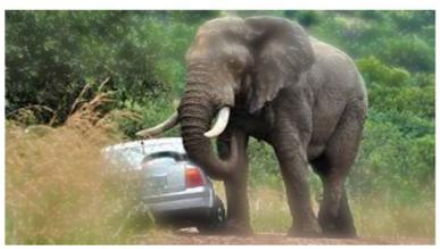

(d)

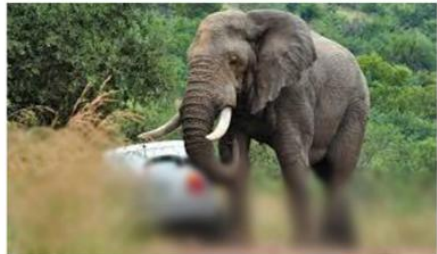

(b)

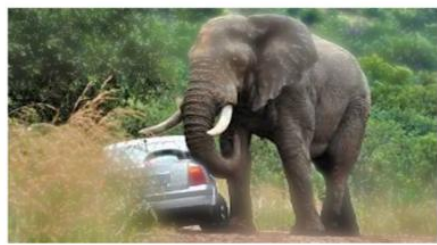

(e)

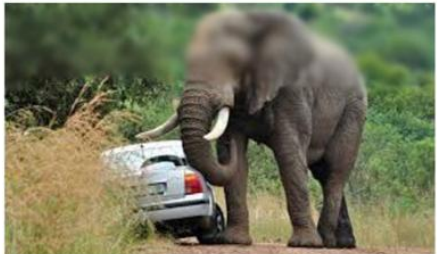

(c)

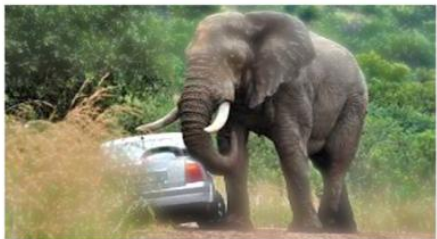

(f)

Fig. 5: Set of Image 1

a. Original Image b \& c. Blurred Image d. Wavelet Fused Image e. PCA Fused Image f. Proposed Hybrid Technique's Fused Image

Table 1: Parametric Comparison of Techniques for Image-1

\begin{tabular}{|c|c|c|c|c|c|c|}
\hline Parameters & $\begin{array}{c}\text { Original } \\
\text { image }\end{array}$ & $\begin{array}{c}\text { Blurred } \\
\text { image: } \mathbf{b}\end{array}$ & $\begin{array}{c}\text { Blurred } \\
\text { image: } \mathbf{c}\end{array}$ & $\begin{array}{c}\text { Wavelet fused } \\
\text { image }\end{array}$ & $\begin{array}{c}\text { PCA fused } \\
\text { image }\end{array}$ & $\begin{array}{c}\text { Proposed } \\
\text { hybrid fusion }\end{array}$ \\
\hline Red contents & 138.282917 & 138.344856 & 138.335896 & $\mathbf{1 4 2 . 1 2 9 8 0 7}$ & $\mathbf{1 3 8 . 3 4 4 0 9 3}$ & $\mathbf{1 4 3 . 8 5 9 0 4 7}$ \\
\hline Green contents & 144.310707 & 144.386101 & 144.378090 & $\mathbf{1 4 8 . 1 5 6 8 2 6}$ & $\mathbf{1 4 4 . 3 8 4 4 0 7}$ & $\mathbf{1 5 0 . 2 5 0 4 6 5}$ \\
\hline Blue contents & 84.508533 & 84.668976 & 84.626976 & $\mathbf{8 8 . 2 2 4 5 3 3}$ & $\mathbf{8 4 . 6 4 7 6 8 6}$ & $\mathbf{9 0 . 0 6 6 6 5 8}$ \\
\hline Entropy & 7.711896 & 7.764397 & 7.776801 & $\mathbf{7 . 8 1 7 3 1 4}$ & $\mathbf{7 . 7 9 4 3 1 3}$ & $\mathbf{7 . 8 2 8 4 2 3}$ \\
\hline
\end{tabular}

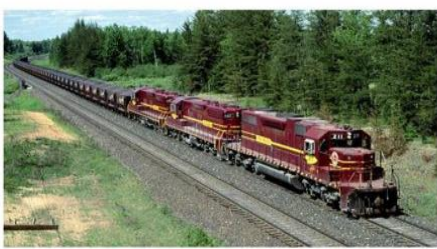

(a)

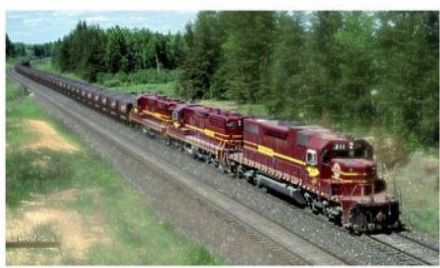

(d)

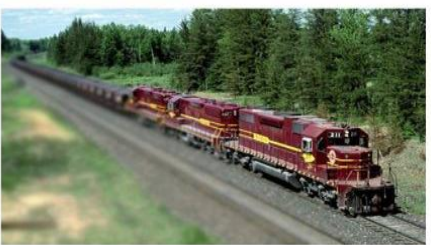

(b)

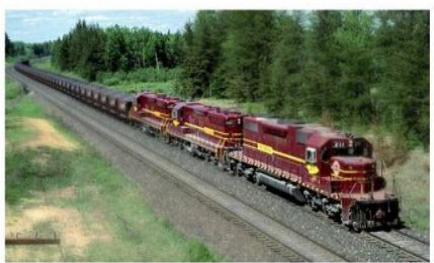

(e)

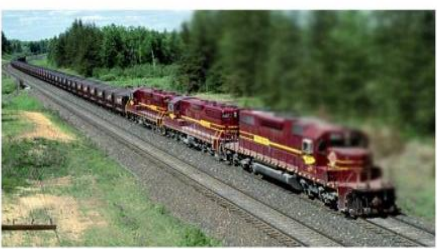

(c)

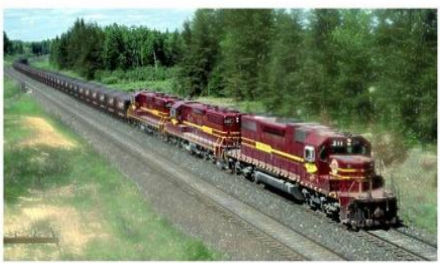

(f)

Fig. 6: Set of Image 2 


\section{a. Original Image b \& c. Blurred Image d. Wavelet Fused Image e. PCA Fused Image f. Proposed Hybrid} Technique's Fused Image

Table 2: Parametric Comparison Techniques for Image-2

\begin{tabular}{|c|c|c|c|c|c|c|}
\hline Parameters & $\begin{array}{c}\text { Original } \\
\text { image }\end{array}$ & $\begin{array}{c}\text { Blurred } \\
\text { image: } \mathbf{b}\end{array}$ & $\begin{array}{c}\text { Blurred } \\
\text { image: } \mathbf{c}\end{array}$ & $\begin{array}{c}\text { Wavelet fused } \\
\text { image }\end{array}$ & $\begin{array}{c}\text { PCA fused } \\
\text { image values }\end{array}$ & $\begin{array}{c}\text { Proposed } \\
\text { hybrid fusion }\end{array}$ \\
\hline Red contents & 133.052135 & 133.141277 & 133.145767 & $\mathbf{1 3 6 . 3 7 6 2 2 1}$ & $\mathbf{1 3 3 . 1 4 3 7 3 0}$ & $\mathbf{1 3 6 . 9 4 6 1 7 3}$ \\
\hline Green contents & 129.297977 & 129.395546 & 129.395008 & $\mathbf{1 3 2 . 8 7 9 1 2 4}$ & $\mathbf{1 2 9 . 3 9 5 2 2 9}$ & $\mathbf{1 3 5 . 1 6 0 6 9 0}$ \\
\hline Blue contents & 113.100773 & 113.199493 & 113.183987 & $\mathbf{1 1 6 . 5 9 8 4 3 1}$ & $\mathbf{1 1 3 . 1 9 4 3 7 8}$ & $\mathbf{1 1 9 . 8 1 6 6 6 6}$ \\
\hline Entropy & 7.616193 & 7.548005 & 7.583164 & $\mathbf{7 . 5 4 6 6 9 2}$ & $\mathbf{7 . 5 4 9 1 6 2}$ & $\mathbf{7 . 5 5 3 5 5 0}$ \\
\hline
\end{tabular}

\section{CONCLUSION AND FUTURE WORK}

The results shows RGB contents, Entropy of the fused image using hybrid technique is more than other technique's fused image. Since this paper is based on considering the remote sensing application. The higher value of RGB contents of an image gives easily detection of an object. The higher value of entropy implies that the proposed technique's fused image is better than the Wavelet \& PCA fused image as these parameters are used to evaluate the information quantity contained in an image. On the basis of qualitative and quantitative analysis of the obtained results, the proposed method is found to be better than spatial domain fusion method (PCA fusion) and transform domain fusion methods (Wavelet transforms).

These hybrid technique can be implemented on various parameters like: Mean Square Error (MSE), Peak Signal to Noise Ratio (PSNR), Bit Error Rate, Root Mean Square Error (RMSE), Percentage Fit Error (PFE), Spatial Frequency, Measure of Structural Similarity (SSIM), and Mutual information (MI), etc. and may provide better results based on these parameters.

\section{REFERENCES}

[1] S.John Nisha Anita, C.John Moses, "Survey On Pixel Level Image Fusion Technique", IEEE International Conference on Emerging Trends in Computing, Communication and Nanotechnology, pp.141-145, March 2013.

[2] Mohamed R. Metwalli, Ayman H. Nasr, Osama S. Farag Allah, and S. El-Rabaie "Image Fusion Based on Principal Component Analysis and High-Pass Filter" IEEE International Conference on Computer Engineering \& Systems, 2009. pp. 63-70, December 2009.

[3] V.P.S. Naidu and J.R. Raol, "Pixel-level Image Fusion using Wavelets and Principal Component Analysis", Defence Science Journal, Vol. 58, No. 3, pp. 338-352, May 2008.

[4] H.B. Kekre, Tanuja Saode, Rachana Dhannawat, "Image Fusion Using Kekre's Hybrid Wavelet Transform", IEEE, International Conference on Communication, Information \& Computing Technology (ICCICT), Mumbai, India, October 2011.

[5] Deepak Kumar Sahu, M P Parsai, "Different image fusion techniques-A Critical Review", International Journal of Modern Engineering Research (IJMER), Vol. 2, Issue. 5, pp-4298-4301 ISSN: 2249-6645, September - October 2012.
[6] S. S. Bedi, Rati Khandelwal, "Comprehensive and Comparative Study of Image Fusion Techniques", International Journal of Soft Computing and Engineering (IJSCE) ISSN: 2231-2307, Volume-3, Issue-1, March 2013.

[7] http://en.wikipedia.org/wiki/Principal_components_analys

[8] Ligia Chiorean, Mircea-Florin Vaida, "Medical Image Fusion Based on Discrete Wavelet Transform Using Java Technology", Proceedings of the ITI $200931^{\text {st }}$ International Conference on Information Technology Interfaces, Cavtat, Croatia, June 2009.

[9] Feng Zhu, Zhi Ming Liu,Liu Yang, Wang Mowei, Xu Shi, Jiang Hai Ling, "Fusion of Remote Sensing Data of the ChangBai Mountain Area Based on the Principal Component and Wavelet Transformation", IEEE, 2nd International Conference on Advanced Computer Control (ICACC), Volume 2, pp. 404-408, March 2010.

[10] Haixia Liu, Xia Zhang, "Comparison of Data Fusion Techniques for Beijing-1 Micro-Satellite Images", IEEE, Urban Remote Sensing Joint Event, pp. 1-6, May 2009.

[11] Rajiv Singh, Richa Srivastava, Om Prakash and Ashish Khare, "Mixed scheme based multimodal medical image fusion using Daubechies Complex Wavelet Transform", IEEE/OSA/IAPR International Conference on Informatics, Electronics \& Vision, pp. 304-309, May 2012.

[12] LU Jun, ZHANG Baoming, HE Hong, ZHANG Hongwei, "The high-pass filtering fusion based on GPU", IEEE, $2^{\text {nd }}$ International Symposium on Computer Science and Society, pp. 122-125, July-2011.

[13] LI Xin-zhi, Wang ping, Zang Yan- bin, "Application of SPOT 5 Data Fusion on Investigating the Ecological Environment of Mining Area", IEEE, Urban Remote Sensing Joint Event 2009, pp.1-4, May 2009.

[14] Yan Meng, Xiaoyun Shen, Renbiao Wu, Ling Hefei, "Remote Sensing Image Fusion Using Multi-Wavelet Transform Combined With HPF", International Conference on Multimedia and Expo 2007 IEEE, pp. 1651-1654, July 2007.

[15] Ramy M Bahy, Gouda Salama, Tarek A. Mahmoud, "A No-Reference Blur Metric Guided Fusion Technique for Multi Focus Images", $28^{\text {th }}$ National Radio Science Conference 2011, pp. 1-9, April 2011. 